\title{
Transpiration Driven Electrokinetic Power Generator
}

Tae Gwang Yun ${ }^{\S \dagger}$, Jaehyeong Bae ${ }^{\S \dagger}$, Avner Rothschild ${ }^{\dagger}$ and Il-Doo Kim ${ }^{\S, *}$

${ }^{\S}$ Department of Materials Science and Engineering, Korea Advanced Institute of Science and Technology (KAIST), 291 Daehak-ro, Yuseong-gu, Daejeon, 34141, Republic of Korea.

Department of Materials Science and Engineering, Technion-Israel Institute of Technology,

Haifa 32000, Israel.

†These authors contributed equally to this work.

*Correspondence: idkim@kaist.ac.kr 


\section{$\underline{\text { Table of contents }}$}

Figure S1. Photograph and SEM image of transpiration driven electrokinteic power generator.

Figure S2. Resistance effect on $I_{S C}$.

Figure S3. Asymmetrical wetting for energy generation.

Figure S4. Temperature dependent $I_{S C}$.

Figure S5. Humidity effect on $I_{S C}$.

Figure S6. Solvent dependent energy generation performance.

Figure S7. Cation dependent energy generation performance.

Figure S8. Rapid energy generation.

Figure S9. XPS results of cotton fabric and Ketjen black.

Figure S10. Resistance dependent energy generation performance.

Figure S11. Configuration of stacked TEPGs.

Figure S12. Schematic illustration of the fabrication of TEPGs and experimental set-up.

Figure S13. IV curves of the TEPGs with various resistances.

Figure S14. Measurement of capillary wicking rate of hydrophilic membrane.

Figure S15. Power generation from TEPG device $(63 \mathrm{k} \Omega)$ with mechanical tensile strain

Figure S16. Power generation from polar aprotic propylene carbonate solutions.

Tables S1. Resistance of TEPGs wetted by $0.75 \mathrm{~mL}$ of various salt solutions.

Video S1. Fabrication and operation of TEPGs.

Video S2. Measurement of voltage from TEPG by dropping $1 \mathrm{~mL}$ of water to fully wet the device.

Video S3. Operation of red LED powered by 30 TEPGs (six series connection of five stacked TEPGs).

Video S4. Operation of electric fan by using the supercapacitor charged by 9 TEPGs (three series connection of three stacked TEPGs). 

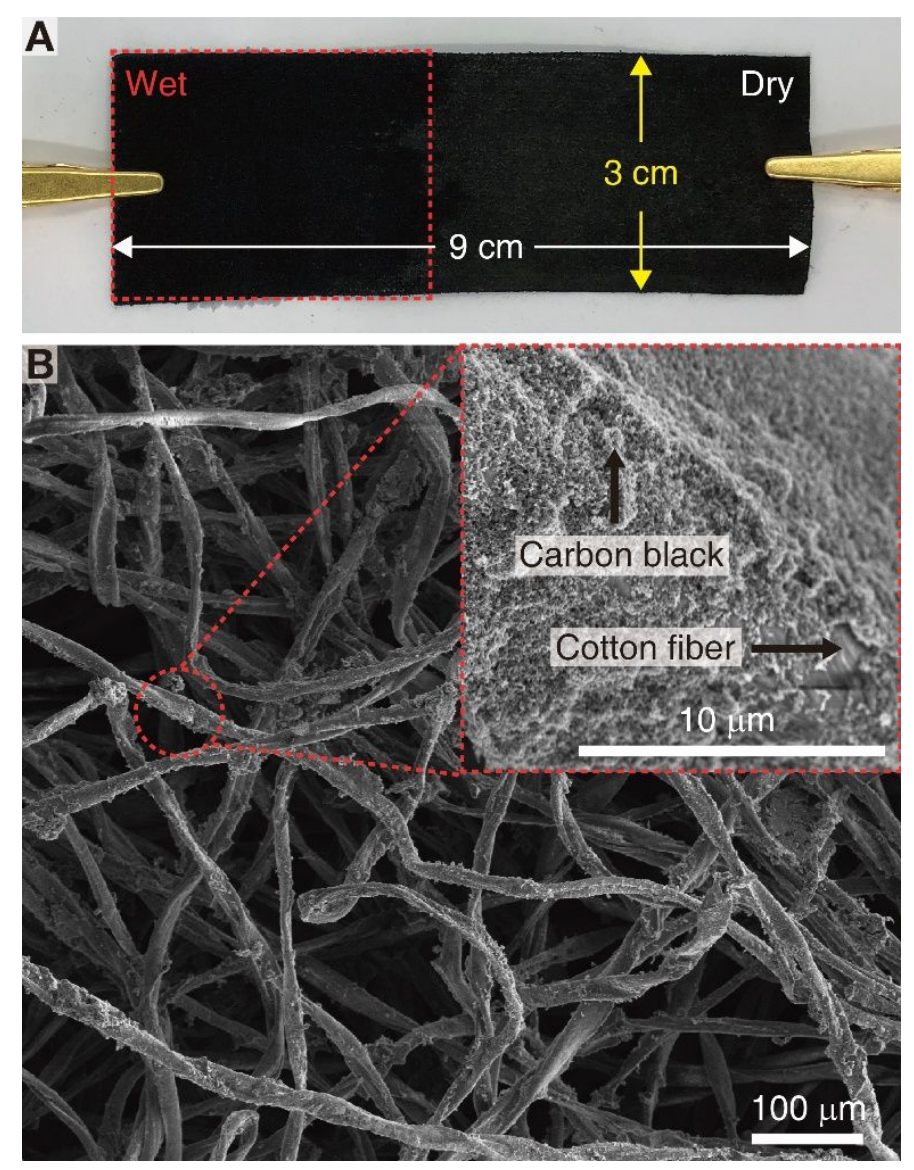

Figure S1. (A) Photograph of transpiration driven electrokinetic power generator (TEPG) composed of carbon black coated cotton fabric. (B) Scanning electron microscope (SEM) image and enlarged SEM image (inset) of the carbon black coated cotton fabric. 

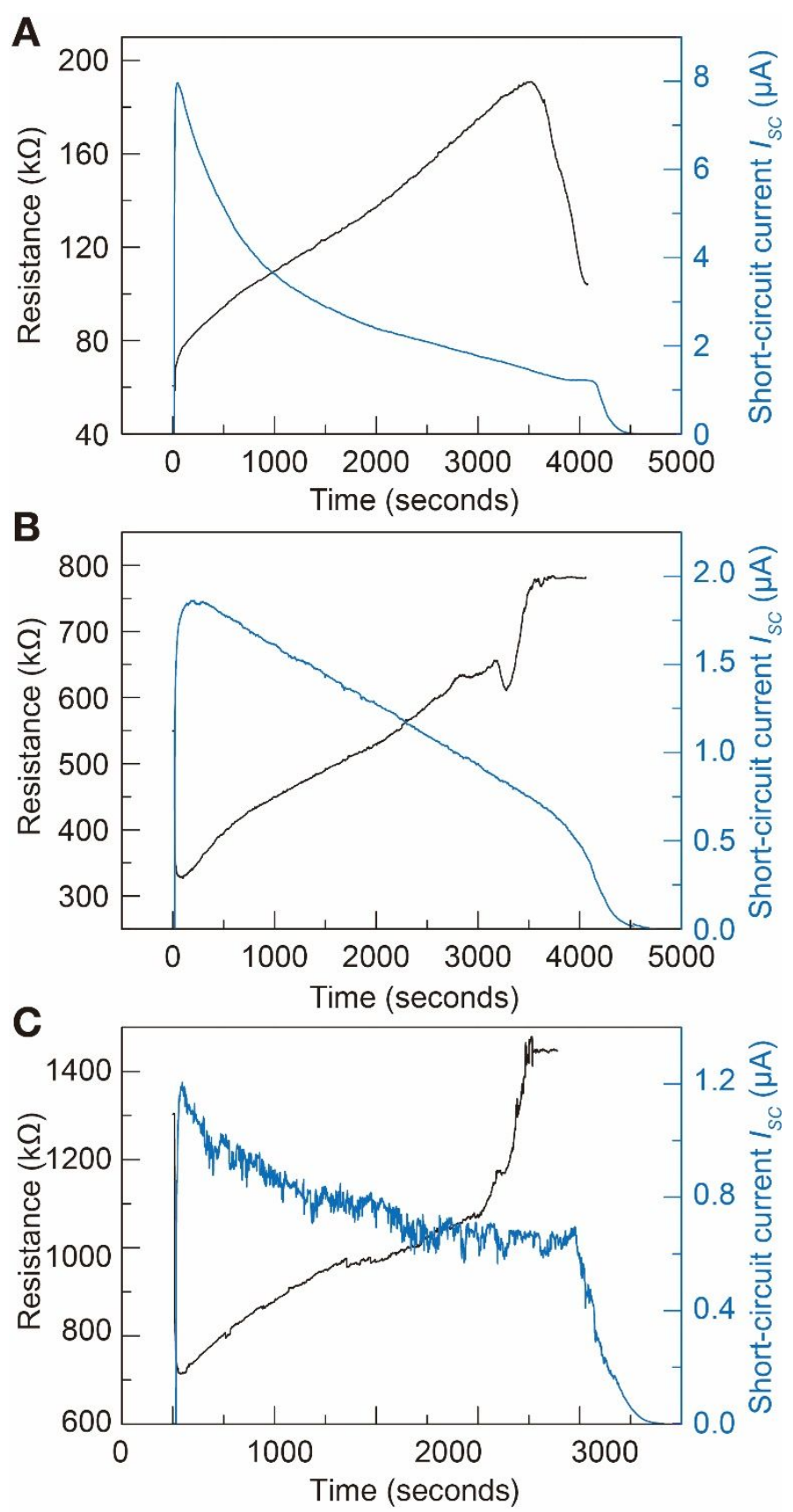

Figure S2. Measured profiles of resistance and $I_{S C}$ during the operation of TEPG. (A), (B), and (C) correspond to the TEPG with initial resistance of $63 \mathrm{k} \Omega, 550 \mathrm{k} \Omega$, and $1.3 \mathrm{M} \Omega$, respectively. 


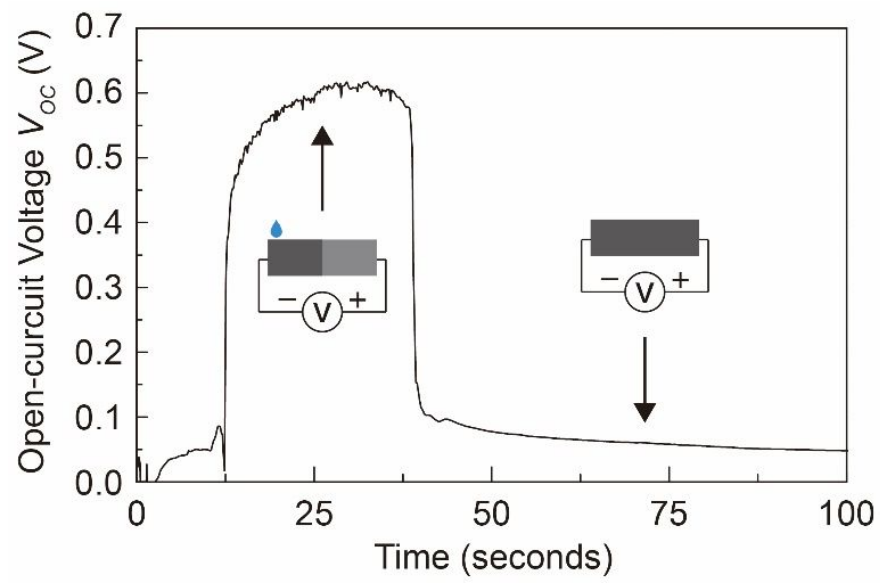

Figure S3. Measured $V_{O C}$ profiles by dropping $1 \mathrm{~mL}$ of water on TEPG. TEPG was thoroughly wetted at 40 seconds and afterwards $V_{O C}$ dropped rapidly. 


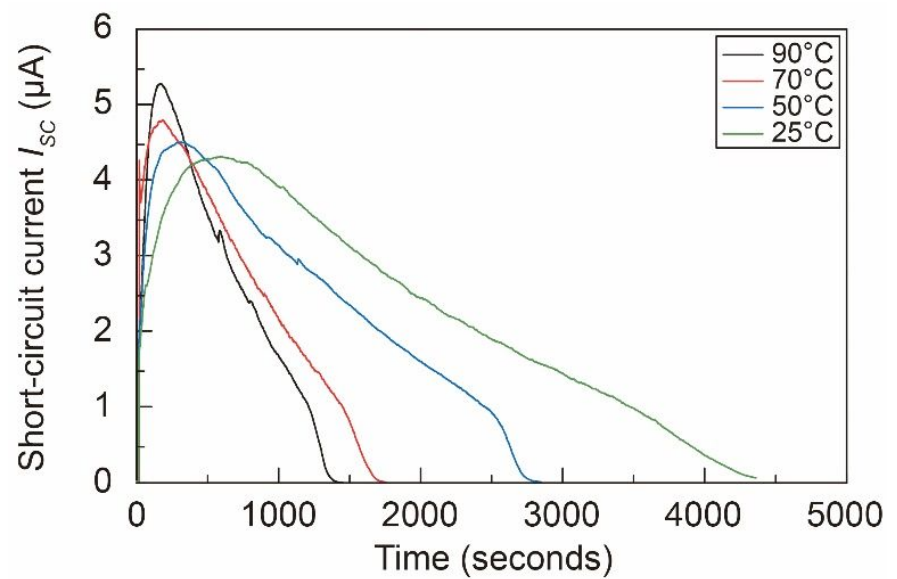

Figure S4. Measured $I_{S C}$ profiles by dropping $0.25 \mathrm{~mL}$ of water on a TEPG at various temperature (resistance of $31 \mathrm{k} \Omega$ ). 


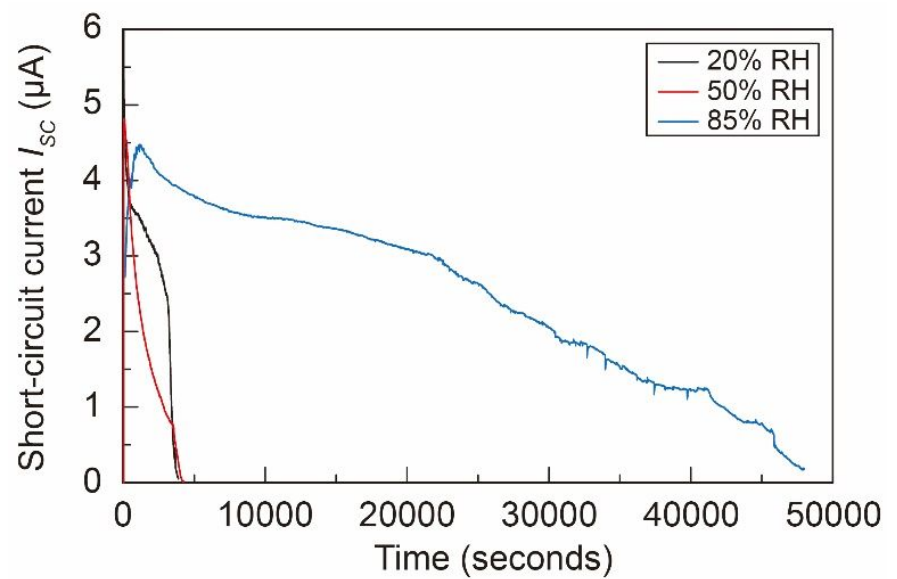

Figure S5. Measured $I_{S C}$ profiles by dropping $0.25 \mathrm{~mL}$ of water on a TEPG at various humidity (resistance of $31 \mathrm{k} \Omega$ ). 


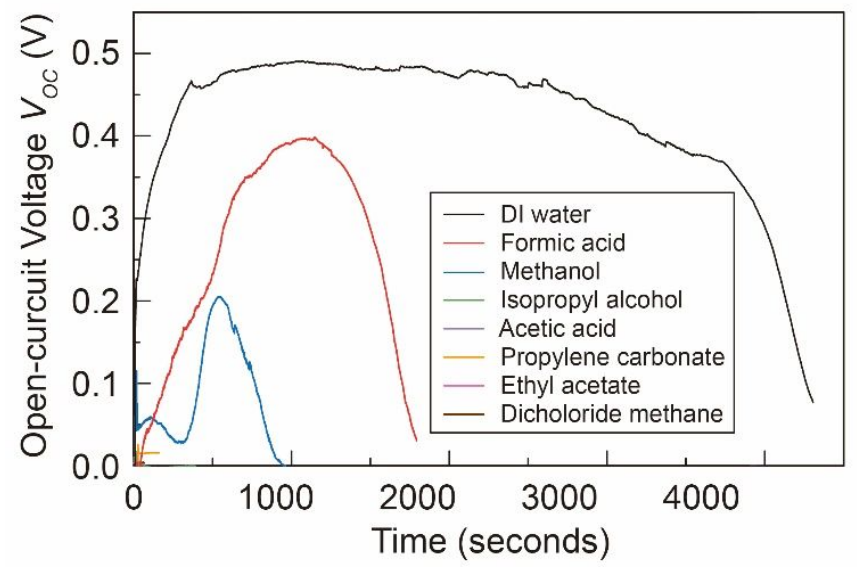

Figure S6. Measured $V_{O C}$ profiles by dropping $0.25 \mathrm{~mL}$ of various liquidson a TEPG (resistance of $270 \mathrm{k} \Omega$ ). 

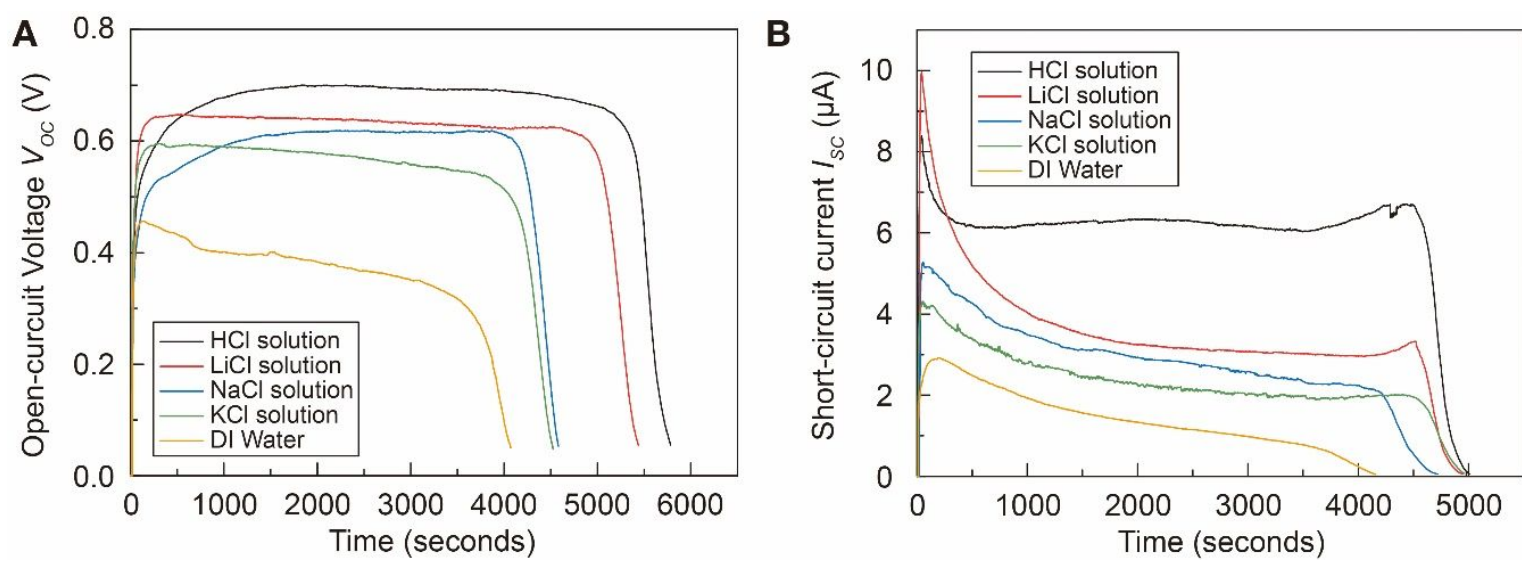

Figure S7. (A) Measured $V_{O C}$ profiles by dropping $0.25 \mathrm{~mL}$ of various salt solution on a TEPG (resistance of $220 \mathrm{k} \Omega$ ). (B) Measured $I_{S C}$ profiles by dropping $0.25 \mathrm{~mL}$ of various salt solution on a TEPG (resistance of $220 \mathrm{k} \Omega$ ). The concentration of salt solutions was $0.1 \mathrm{M}$. 


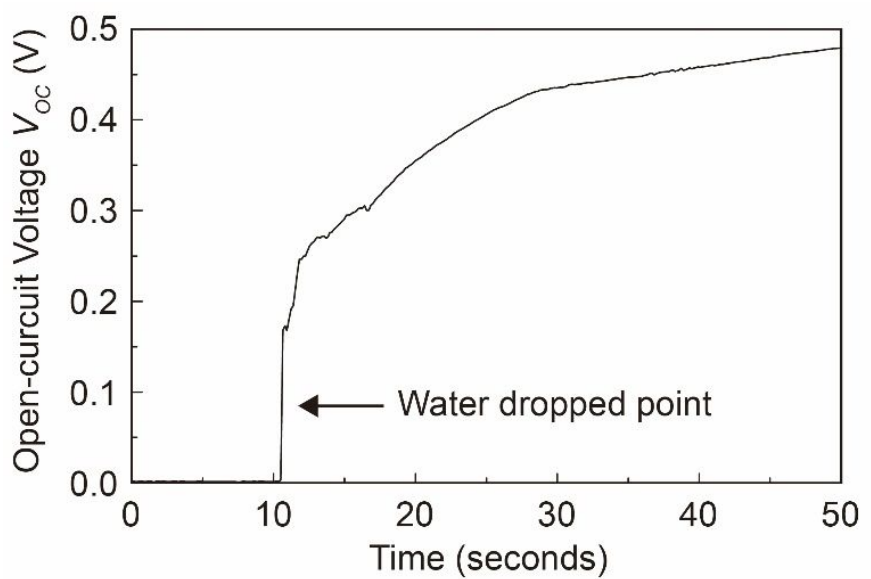

Figure S8. Measured $V_{O C}$ profiles right after dropping water on a TEPG. 
A

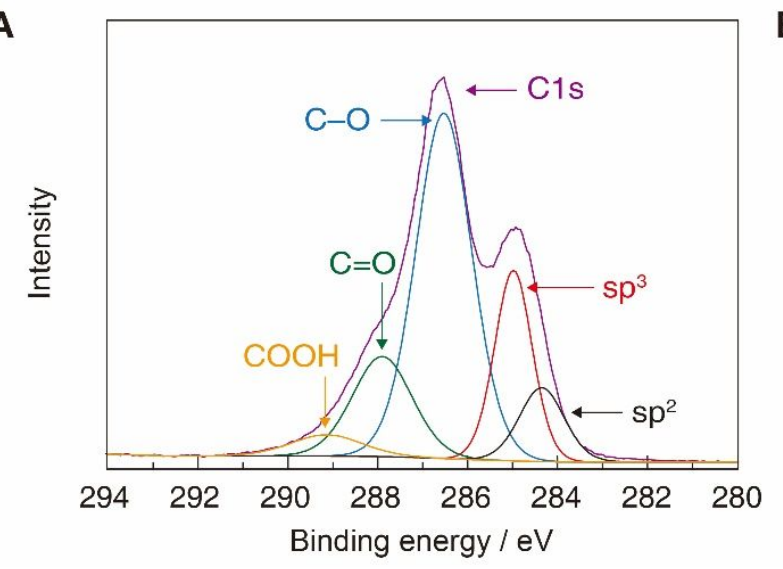

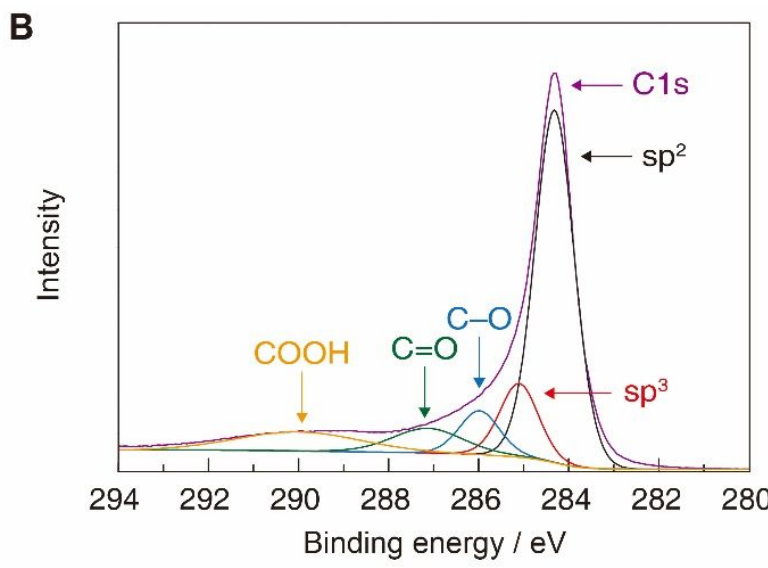

Figure S9. (A) C1s X-ray photoelectron spectroscopy (XPS) result of cotton fabric. (B) C1s XPS result of Ketjen black.

Note: XPS analysis was performed to figure out functional groups formed on the surface of commercially available cotton fabric and Ketjen black. For cotton fabric, the peaks of hybridized carbon atoms were found at $284.3\left(\mathrm{sp}^{3}\right)$ and $285.0 \mathrm{eV}\left(\mathrm{sp}^{2}\right)$. The peaks of oxygen functional group were found at $286.5(\mathrm{C}-\mathrm{O}), 287.9(\mathrm{C}=\mathrm{O})$, and $289.1 \mathrm{eV}(\mathrm{COOH})$. For Ketjen black, the peaks of hybridized carbon atoms were found at $284.3\left(\mathrm{sp}^{3}\right)$ and $285.3 \mathrm{eV}\left(\mathrm{sp}^{2}\right)$. The peaks of oxygen functional group were found at $286.1(\mathrm{C}-\mathrm{O}), 287.2(\mathrm{C}=\mathrm{O})$, and $289.9 \mathrm{eV}$ $(\mathrm{COOH})$. 

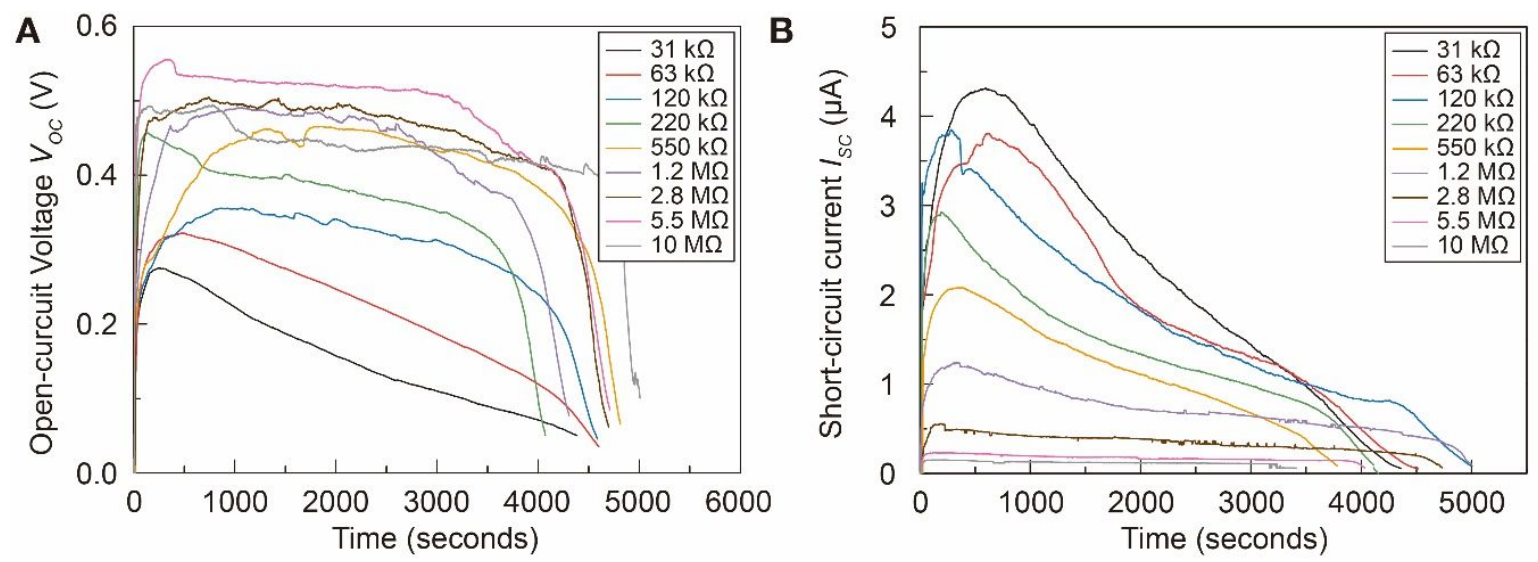

Figure S10. (A) Measured $V_{O C}$ profiles by dropping $0.25 \mathrm{~mL}$ of water on various resistances of TEPG. (B) Measured $I_{S C}$ profiles by dropping $0.25 \mathrm{~mL}$ of water on various resistances of TEPG. 

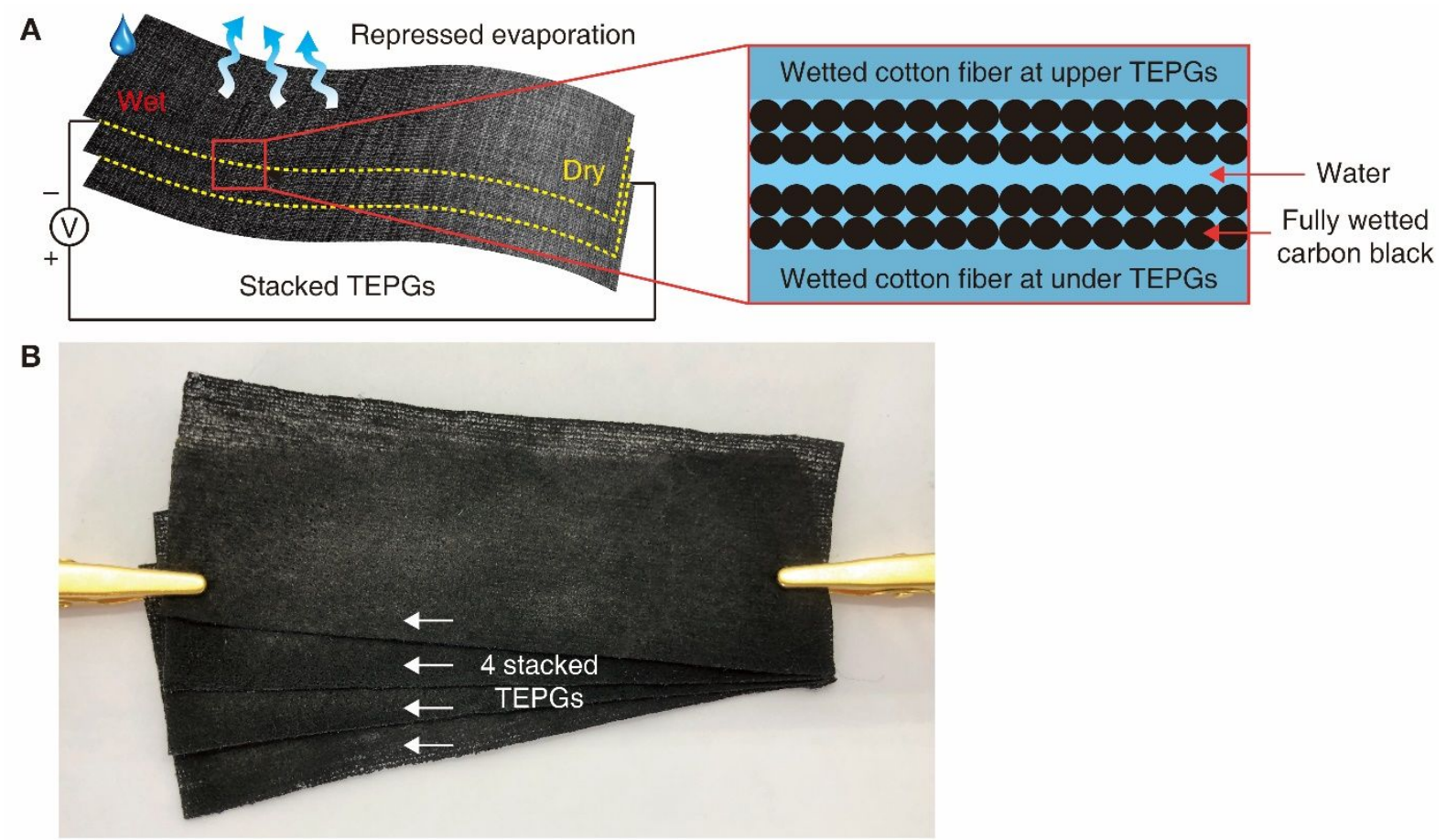

Figure S11. (A) Schematic illustration of stacked TEPGs and the configuration of carbon black layers in-between two TEPGs. (B) Photographs of stacked four-layer of TEPGs. 
A Cotton Ketjen black coated fabric cotton fabric

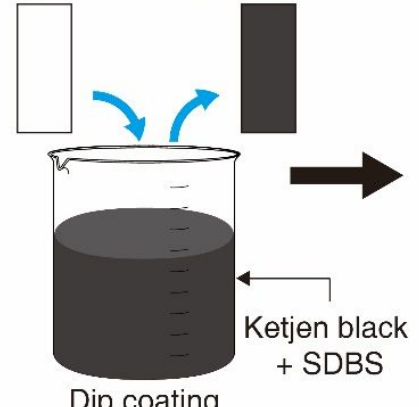

Dip coating
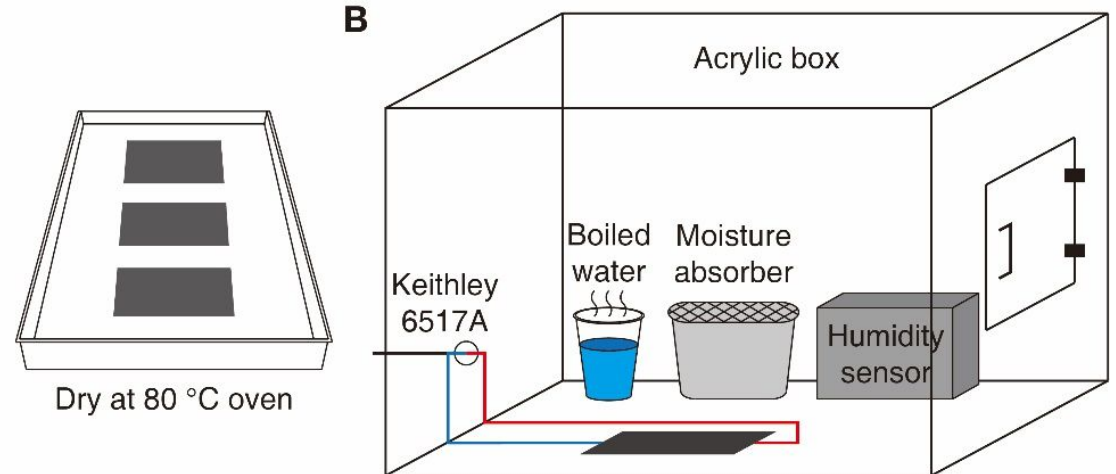

Figure S12. (A) Schematic illustration of the fabrication of TEPGs. (B) Schematic illustration of experimental set-up for humidity control. The humidity was controlled from $20 \% \mathrm{RH}$ to $85 \%$ RH by placing either moisture absorber or boiled water in acrylic box. 


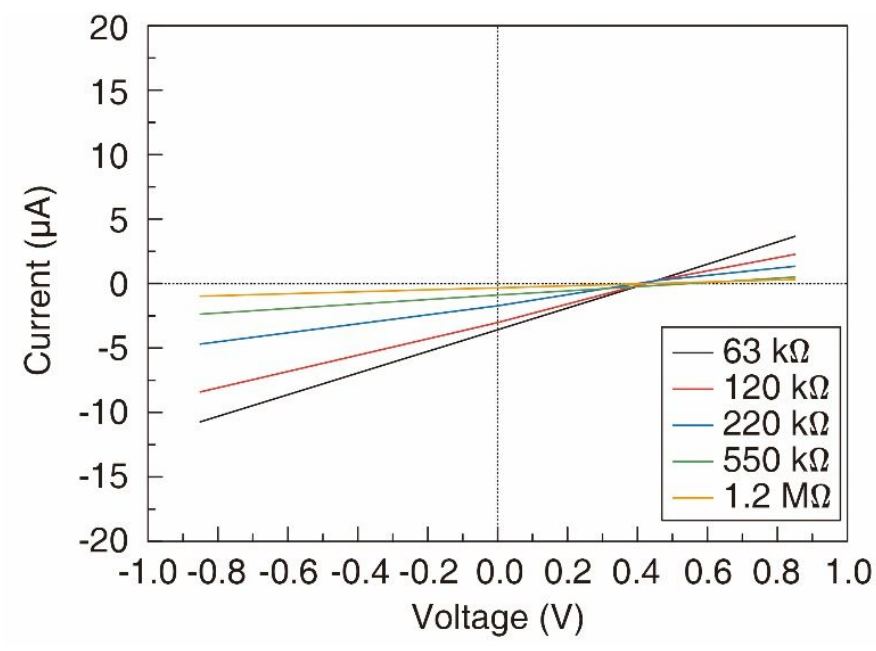

Figure S13. IV curves of the TEPGs with various resistances. The IV curves were measured by applying voltage source with the scan rate of $10 \mathrm{mV} / \mathrm{s}$ at a voltage ranging from $-0.85 \mathrm{~V}$ to $0.85 \mathrm{~V}$. Based on the IV curves, TEPG exhibits Ohmic contact behavior. 


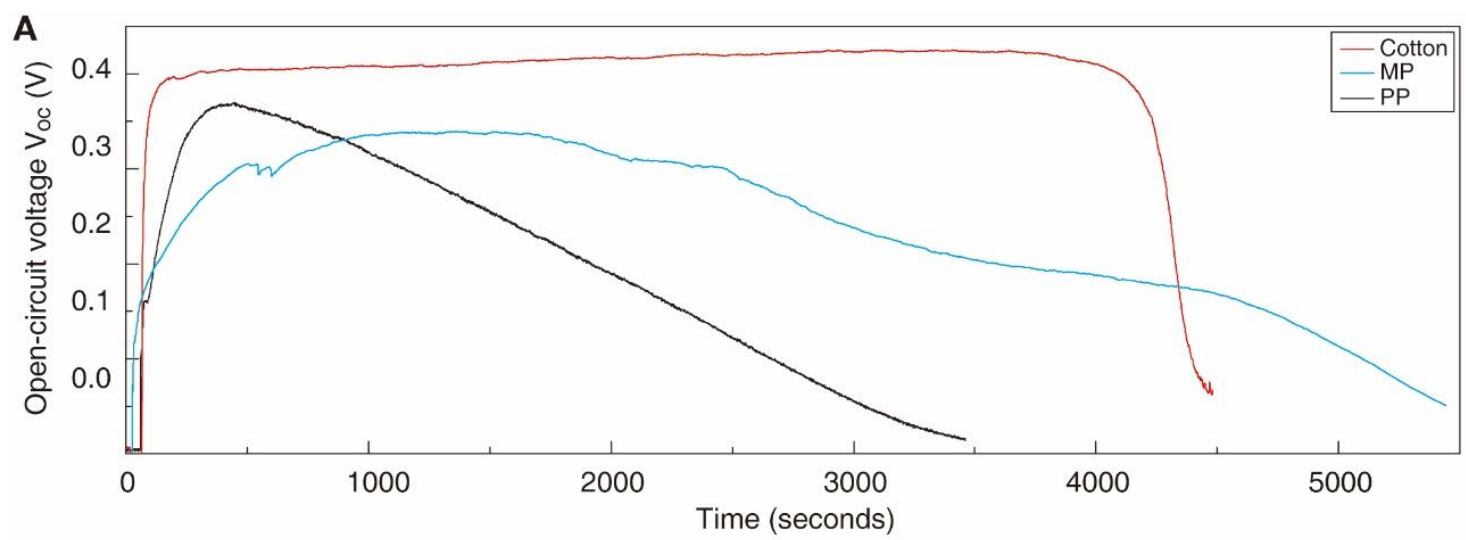

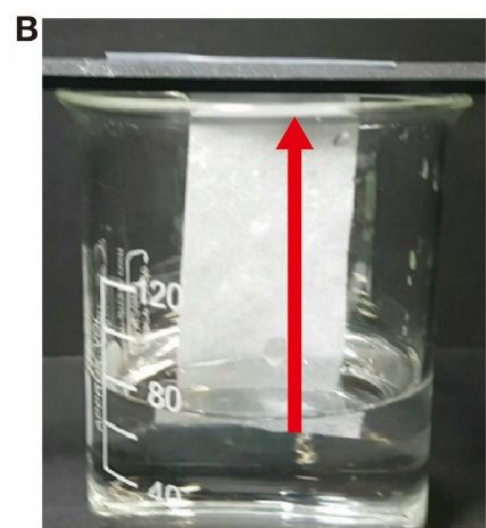

Cotton

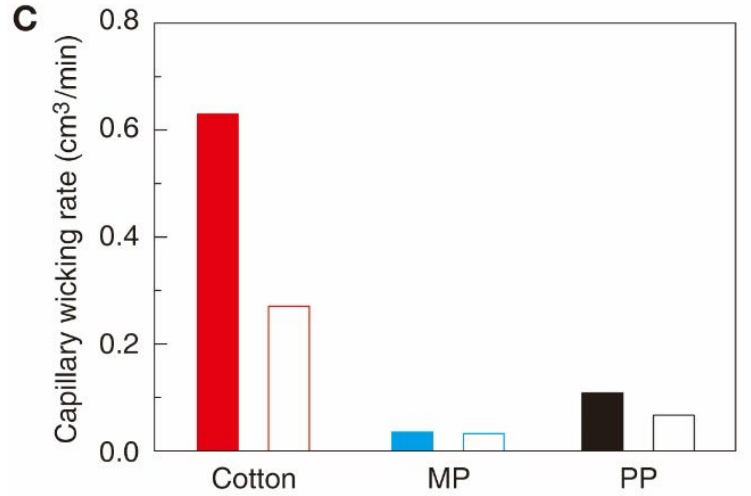

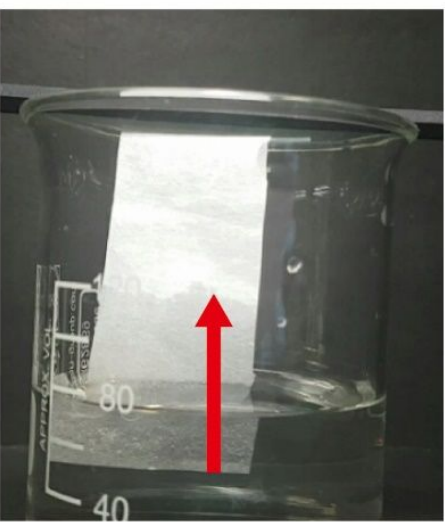

Mulberry paper

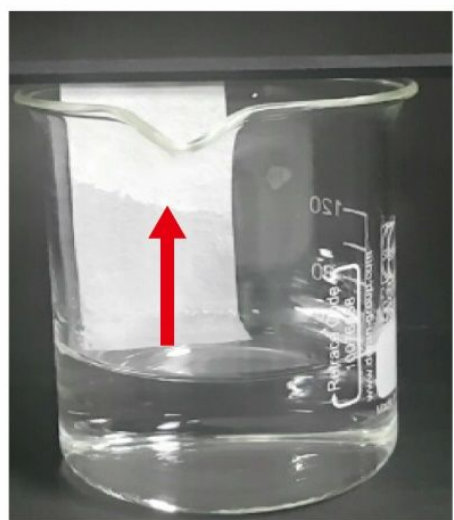

Polypropylene membrane

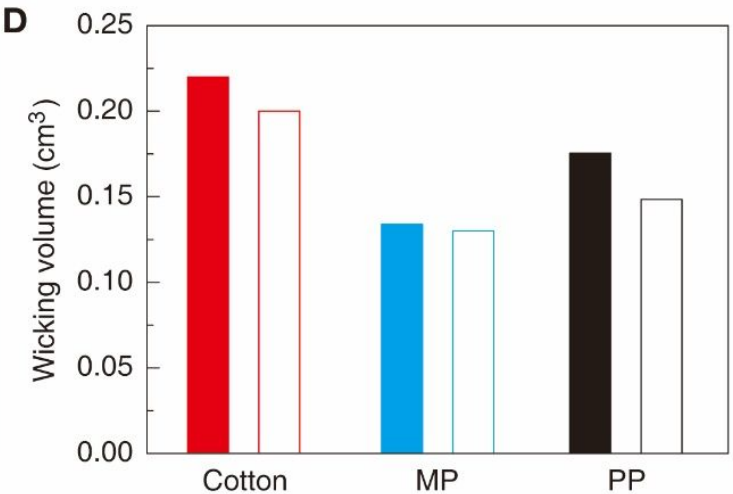

Figure. S14. (A) Measured $V_{o c}$ profiles by dropping $0.25 \mathrm{ml}$ of water on Ketjen black coated cotton fabric, mulberry paper (MP), and hydrophilic polypropylene membrane (PP); the carbon loading was fixed around $6 \mathrm{mg}$. (B) Photograph of the method of capillary wicking rate. (C) Capillary wicking rate of Ketjen black coated cotton fabric, mulberry paper, and hydrophilic polypropylene membrane (colored column) and those of pristine (empty column). (D) Water wicking volume of Ketjen black coated cotton fabric, mulberry paper, and hydrophilic polypropylene membrane (colored column) and those of pristine (empty column). 


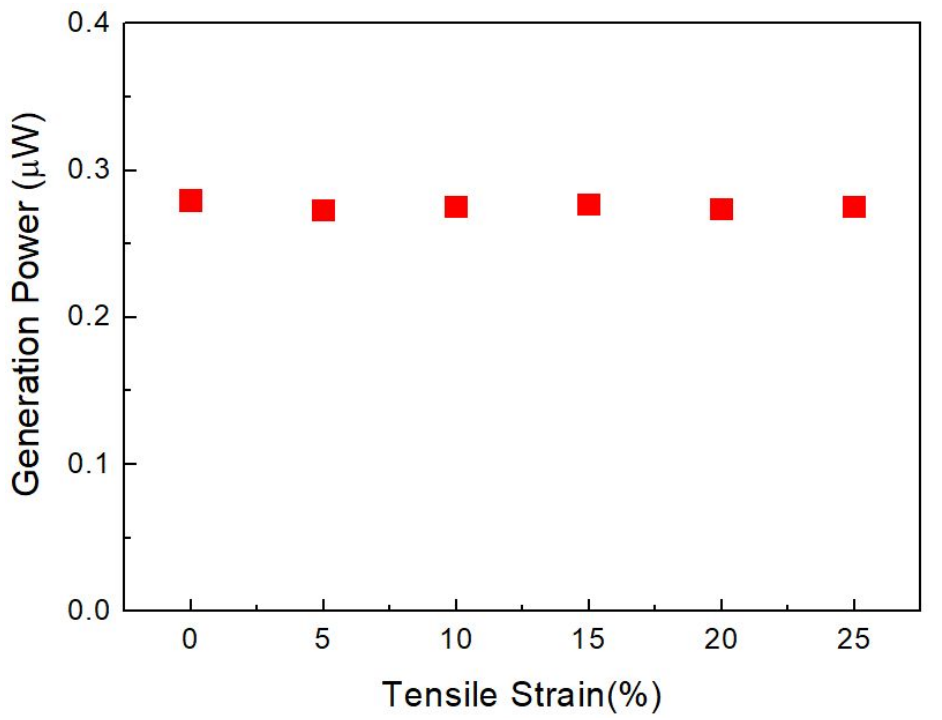

Figure S15. Stable power generation from TEPG device $(63 \mathrm{k} \Omega)$ with mechanical tensile strain. 

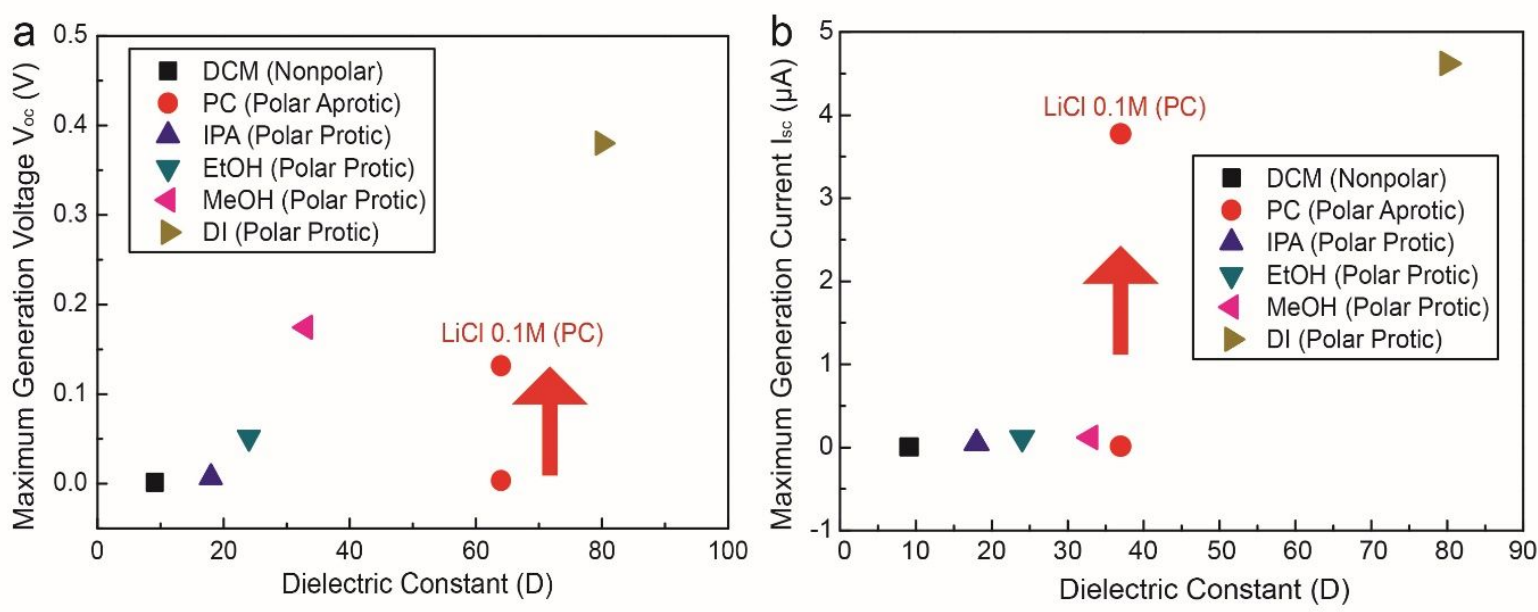

Figure S16. Comparison of the maximum Voc, Isc values by dropping $0.25 \mathrm{~mL}$ of various types and polarity of liquids on TEPG (resistance of $1.3 \mathrm{M} \Omega$ ). Pure propylene carbonate did not generate both voltage and current; however, $0.1 \mathrm{M}$ of $\mathrm{LiCl}$ added propylene carbonate solution could generate voltage and current, showing that ions play critical role in energy generation in TEPG. 
Tables S1. Resistance of TEPGs wetted by $0.75 \mathrm{~mL}$ of various salt solutions. This result shows that addition of inorganic salt decreases the resistance of the device.

\begin{tabular}{lll}
\hline & Resistance of TEPG in dry & $\begin{array}{l}\text { Resistance of TEPG after } \\
\text { wetting }\end{array}$ \\
\hline DI water & $18.65 \mathrm{k} \Omega$ & $91.3 \mathrm{k} \Omega$ \\
\hline $0.1 \mathrm{M}$ of LiCl solution & $21.70 \mathrm{k} \Omega$ & $8.05 \mathrm{k} \Omega$ \\
\hline $0.1 \mathrm{M}$ of NaCl solution & $18.60 \mathrm{k} \Omega$ & $6.18 \mathrm{k} \Omega$ \\
\hline $0.1 \mathrm{M}$ of KCl solution & $20.02 \mathrm{k} \Omega$ & $10.29 \mathrm{k} \Omega$ \\
\hline
\end{tabular}


Video S1. Fabrication and operation of TEPGs.

Video S2. Measurement of voltage from TEPG by dropping $1 \mathrm{~mL}$ of water to fully wet the device.

Video S3. Operation of red LED powered by 30 TEPGs (six series connection of five stacked TEPGs).

Video S4. Operation of electric fan by using the supercapacitor charged by 9 TEPGs (three series connection of three stacked TEPGs). 


\section{Derivation of equations}

\section{Effect of the internal resistance of TEPG on $V_{O C}$ and $\boldsymbol{I}_{S C}$.}

In TEPG device, higher internal resistance exhibited higher $V_{O C}$ and lower $I_{S C}$ (Figure $3 \mathrm{~A}$ ). To figure out the trade-off relationship of internal resistance on $V_{O C}$ and $I_{S C}$, we analyzed the working TEPG as in Figure 3B. The internal resistance can be analyzed into $R_{w i}$ (the innermost carbon layer attached to cotton in the wet region), $R_{w e}$ (excess exterior carbon layer in wet region), and $R_{d}$ (carbon coated on the dry region). As innermost carbon layer is in the proximity of cotton fabric, $R_{w i}$ would develop better electrical double layer for $V_{E D L}$, and produce pseudostreaming current $I_{P S T}$. $R_{d}$ is connected in series with wetted carbon regions. If the TEPG generates current, the TEPG will lose voltage over the $R_{d} . R_{w e}$ refers to as shunt resistance that is connected in parallel with $R_{w i}$. Excess exterior carbon layer above the innermost carbon layer provides the alternative path for the generated current. Low shunt resistance means that a large fragment of the current prefers to travel through the shunt.

TEPG is desirable to have the $R_{d}$ as small as possible and the $R_{w e}$ as large as possible to acquire ideal emanated $V_{E D L}$ and $I_{P S T}$ from TEPG. However, the fabrication method of TEPG only allows concurrent control of $R_{w e}$ and $R_{d}$. Reduction of internal resistance, by dipping cotton fabric into carbon black ink multiple times, decreases both $R_{w e}$ and $R_{d}$. The series and shunt resistance result in a more complicated expression for the $V_{o c}$ and $I_{s c}$.

The voltage at the terminal is the voltage of an ideal $V_{E D L}$ minus the voltage lost over the series resistances $\left(R_{w i}\right.$ and $\left.R_{d}\right)$.

$$
V=V_{E D L}-I_{P S T} R_{w i}-I R_{d}
$$

The current at the terminal is the $I_{P S T}$ current minus the shunt current $\left(I_{s h}\right)$ leaking through the shunt resistance.

$$
I=I_{P S T}-I_{S h}
$$

The shunt current is the voltage of an ideal $V_{E D L}$ potential divided by the shunt resistance.

$$
I_{s h}=\frac{V_{E D L}-I_{P S T} R_{w i}}{R_{w e}}
$$

Combining equation (2) and equation (3)

$$
I=I_{P S T}-\frac{V_{E D L}-I_{P S T} R_{w i}}{R_{w e}}
$$

Rearranging equation (4)

$$
I_{P S T}=\frac{V_{E D L}+I R_{w e}}{R_{w i}+R_{w e}}
$$


Combining equation (1) and equation (5)

$$
V=V_{E D L}-\frac{V_{E D L}+I R_{w e}}{R_{w i}+R_{w e}} R_{w i}-I R_{d}
$$

By the definition of open-circuit voltage, the current at the terminal is zero. Therefore, equation (6) becomes

$$
V_{O C}=V_{E D L}-\frac{V_{E D L} R_{w i}}{R_{w i}+R_{w e}}=V_{E D L}\left(1-\frac{R_{w i}}{R_{w i}+R_{w e}}\right)
$$

From equation (7), we can see that reduction of the internal resistance increases the fraction in the parenthesis, which results in decrease of $V_{O C}$.

Rearranging equation (5)

$$
V_{E D L}=I_{P S T}\left(R_{w i}+R_{w e}\right)-I R_{w e}
$$

Combining equation (1) and equation (8)

$$
I=\frac{I_{P S T} R_{w e}-V}{R_{w e}+R_{d}}
$$

By the definition of short-circuit current, the voltage at the terminal is zero. Therefore, equation (9) becomes

$$
I_{S C}=\frac{I_{P S T} R_{w e}}{R_{w e}+R_{d}}=\frac{I_{P S T}}{1+\frac{R_{d}}{R_{w e}}}
$$

From equation (10), it is hard to see the resistance effect on $I_{S C}$.

Let us assume $V_{E D L}$ and $I_{P S T}$ is constant, as origin of $V_{E D L}$ and $I_{P S T}$ is not related to amount of carbon black.

Combining equation (4) and equation (10)

$$
I_{S C}=\frac{V_{E D L}\left(1-\frac{R_{w i}}{R_{w i}+R_{w e}}\right)}{\frac{R_{w i} R_{w e}}{R_{w i}+R_{w e}}+R_{d}}=\frac{V_{E D L}}{R_{w i}+R_{d}\left(1+\frac{R_{w i}}{R_{w e}}\right)}
$$


From equation (11), we can see that reduction of the internal resistance decreases the denominator, which results in increase of $I_{S C}$.

\section{Effect of the internal resistance of TEPG on $\boldsymbol{P}_{M A X}$.}

TEPG exhibits Ohmic contact behavior, which means that the maximum power generated in specific resistance is one-half of $V_{O C}$ multiple by one-half of $I_{S C}$ (Figure S13).

Using the equation (7) and (9)

$$
\begin{gathered}
P_{M A X}=\frac{1}{4} V_{O C} I_{S C}=\frac{1}{4} \cdot V_{E D L}\left(1-\frac{R_{w i}}{R_{w i}+R_{w e}}\right) \cdot \frac{V_{E D L}}{R_{w i}+R_{d}\left(1+\frac{R_{w i}}{R_{w e}}\right)} \\
=\frac{V_{E D L}^{2}}{4} \cdot \frac{R_{w e}}{R_{w i}+R_{w e}} \cdot \frac{1}{R_{w i}+R_{d}+\frac{R_{w i} R_{d}}{R_{w e}}}
\end{gathered}
$$

Depending on the loading amount of carbon black, the configuration of the device can be considered into two situations. If the loading amount of carbon black was too small, carbon black would not form a monolayer (the innermost carbon layer) on the cotton fabric; therefore, no carbon is working for shunt resistance $\left(R_{w e}\right)$.

In this situation, the boundary conditions are

$$
\begin{gathered}
R_{\text {monolayer }}<R_{\text {wet total }}=\frac{1}{\frac{1}{R_{w i}}+\frac{1}{R_{w e}}}<\infty, \\
R_{w e}=\infty, R_{\text {monolayer }}<R_{w i}<\infty
\end{gathered}
$$

If we assume that dry carbon and wet carbon have following relationship,

$$
R_{d}=\alpha R_{w i}
$$

The equation (10) can be expressed into

$$
\begin{gathered}
P_{M A X}=\frac{V_{E D L}^{2}}{4} \cdot \frac{R_{w e}}{R_{w i}+R_{w e}} \cdot \frac{1}{R_{w i}+R_{d}+\frac{R_{w i} R_{d}}{R_{w e}}} \\
=\frac{V_{E D L}^{2}}{4} \cdot \frac{\infty}{R_{w i}+\infty} \cdot \frac{1}{R_{w i}+\alpha R_{w i}+\frac{\alpha R_{w i}^{2}}{\infty}} \approx \frac{V_{E D L}^{2}}{4} \cdot \frac{1}{(1+\alpha) R_{w i}}
\end{gathered}
$$


At the low loading ranges, reduction of the internal resistance $\left(R_{w i}\right)$ increases $P_{M A X}$ by means of adding more carbon black. As the $P_{M A X}$ does not have mathematical maximum value in the boundary condition, we need to consider the situation that the loading amount of carbon black is large enough that there are excess carbons after forming monolayer of carbon black on the cotton fiber.

In the second situation, the boundary conditions are

$$
\begin{gathered}
0<R_{\text {wet total }}=\frac{1}{\frac{1}{R_{w i}}+\frac{1}{R_{w e}}}<R_{\text {monolayer }} \\
R_{w i}=R_{\text {monolayer }}=\text { constant }, 0<R_{w e}<\infty
\end{gathered}
$$

If we assume that dry carbon and wet carbon has following relationship,

$$
R_{d}=\beta R_{w e}
$$

The equation (10) can be expressed into

$$
\begin{aligned}
& P_{M A X}=\frac{V_{E D L}^{2}}{4} \cdot \frac{R_{w e}}{R_{w i}+R_{w e}} \cdot \frac{1}{R_{w i}+R_{d}+\frac{R_{w i} R_{d}}{R_{w e}}} \\
& =\frac{V_{E D L}^{2}}{4} \cdot \frac{R_{w e}}{\beta R_{w e}^{2}+(1+2 \beta) R_{w i} R_{w e}+(1+\beta) R_{w i}^{2}}
\end{aligned}
$$

To find the resistance that gives mathematical maximum value of $P_{M A X}$, taking the derivatives of $P_{M A X}$ with respect to $R_{w e}$.

$$
\frac{d P}{d R_{w e}}=\frac{V_{E D L}^{2}}{4} \cdot \frac{(1+\beta) R_{w i}^{2}-\beta R_{w e}^{2}}{\left[\beta R_{w e}^{2}+(1+2 \beta) R_{w i} R_{w e}+(1+\beta) R_{w i}^{2}\right]^{2}}
$$

The derivative is zero at:

$$
R_{w i}=\sqrt{\frac{1+\beta}{\beta}} R_{w e}
$$

If the $\beta$ is large enough, bilayer of carbon blacks on cotton fabric is the configuration for the highest $P_{M A X}$. 